\title{
System Spectral Efficiency and Stability of 3G Networks: A Comparative Study
}

\author{
Yuehong Gao ${ }^{\dagger \ddagger}$, Xin Zhang ${ }^{\dagger}$, Yuming Jiang ${ }^{\ddagger}$ and Jeong-woo Cho ${ }^{\ddagger}$ \\ ${ }^{\dagger}$ Wireless Theories and Technologies (WT\&T) Lab. \\ Beijing University of Posts and Telecommunications (BUPT), 100876 Beijing, China \\ ${ }^{\ddagger}$ Centre for Quantifiable Quality of Service in Communication Systems (Q2S) \\ Norwegian University of Science and Technology (NTNU), NO-7491 Trondheim, Norway
}

\begin{abstract}
CDMA2000, WCDMA and WiMAX are three widely used $3 G$ technologies. Since they share the same goal, which is to provide broader coverage and higher throughput in 3G networks, an impartial comparison of their performance is indispensable. However, they are based on different design principles and methodologies, which make the comparison challenging. This paper presents a comparative study of these technologies, with focus on system spectral efficiency and stability in 3G networks. Specifically, the paper presents a framework for the comparison based on the common set of configurations adopted by these technologies, which include channel models, system parameters and key algorithms. Through extensive simulations, the system spectral efficiency and stability of CDMA2000 1x EVDO Rev.A, WCDMA HSDPA/HSUPA and Mobile WiMAX are compared. It is found that while WiMAX can provide highest throughput, the two CDMA-based technologies achieve higher system spectral efficiency, especially on the downlink. Regarding system stability, it is observed that CDMA2000 1x EV-DO Rev.A can operate under higher interference levels than WCDMA HSDPA/HSUPA and Mobile WiMAX. In addition, the comparison on system spectral efficiency between CDMA2000, WCDMA and WiMAX is also conducted when relevant enhanced technologies, i.e., MIMO and interference cancelation, are adopted. We believe that our work will serve as a cornerstone for a fair comparison between the various technologies for prospective $3 \mathrm{G}$ networks.
\end{abstract}

\section{INTRODUCTION}

Wireless communication and networking technologies have evolved rapidly. To date, two Code Division Multiple Access (CDMA) variants are widely used in 3rd generation (3G) mobile networks, which are CDMA2000 and WCDMA. In addition, WiMAX is an emerging technology for $3 \mathrm{G}$ networks. However, there are significant differences among these technologies. For example, while CDMA2000 and WCDMA use Frequency Division Duplex (FDD) solutions based on 1.25 $\mathrm{MHz}$ and $5 \mathrm{MHz}$ bandwidth respectively, the current version of WiMAX uses Orthogonal Frequency Division Multiplex (OFDM) technique with Time Division Duplex (TDD) mode and a relatively wide bandwidth, typically $10 \mathrm{MHz}$.

In spite of these great differences in specification, these technologies still share the same goal, i.e., to provide broader coverage and higher throughput under mobile wireless access environment. This motivates our interest in comparing the system performance of these technologies. Considering the scarcity of spectrum and the cost of bandwidth rent, such comparisons are even more critical for network operators who choose the technologies that best suit their needs.

The challenge in performing performance comparison of these technologies is due to that there is rich variety in key techniques used in them. In the literature, many organizations and researchers have published results concerning performance evaluation of these technologies, e.g., [1-3]. However, to the best of our knowledge, there has been only one work [4], which tries to compare the performance of CDMA2000, WCDMA and WiMAX. Their attention was focused on throughput and spectral efficiency. Though they claim that WiMAX significantly outperforms the other two [4], this conclusion is doubtful because the framework used for the comparison is arguable. For example, the channel model used in [4] is the 5-type model proposed in [1], which is not suitable for broad band systems because of different resolution capability on multipath. In addition, the overhead size of WiMAX is fixed to a specific value, e.g., 7 OFDM symbols on the downlink (DL), without providing any convincing reason. Furthermore, a serious pitfall is that many key parameters are not clearly enumerated.

The objective of this paper is to compare the performance of CDMA2000, WCDMA and WiMAX. In addition to system spectral efficiency, the comparison will also focus on system stability. For the comparison, a common set of configurations adopted by these technologies are firstly identified, which include channel models, system parameters and key algorithms. Simulation results are then presented and compared. In order to give a fairer comparison, the simulation platform is calibrated so that the throughput is maximized and at the same time a fairness criterion among users is met. One of our key findings is that the conclusions made in [4] do not hold if all these technologies are compared in the less-biased and more-fair configuration. Specifically we find that while WiMAX can provide highest throughput, the two CDMAbased technologies achieve higher system spectral efficiency, especially on the downlink. Regarding system stability, it is observed that CDMA2000 1x EV-DO Rev.A can operate under higher interference levels than the others. In addition, the comparison on system spectral efficiency is also conducted when relevant enhanced technologies, i.e., MIMO and interference cancelation, are adopted. 
The rest of this paper is organized as following. First, an overview of the three $3 \mathrm{G}$ technologies is presented in Section II, where their key features are compared. Then a framework used for performance comparison is introduced in Section III, which includes the common set of configurations, system parameters and key algorithms. In Section IV, the simulation platform and results under both basic and enhanced techniques are described. Discussion and insight into the observations from the simulation results are also presented in this section. Finally, Section V concludes the paper.

\section{OVERVIEW OF 3G NETWORKS}

This section provides an overview of CDMA, WCDMA and WiMAX. Particular focus is on CDMA2000 1x EV-DO Rev.A, WCDMA HSDPA/HSUPA (HSxPA) and IEEE 802.16e Mobile WiMAX, which are respectively evolutional version of CDMA, WCDMA and WiMAX, which have been widely used for existing $3 \mathrm{G}$ networks.

CDMA2000 1x EV-DO Rev.A [5], denoted by EV-DO Rev.A in this paper, is an enhanced version to CDMA2000 1x EV-DO Rel.0. In EV-DO Rev.A, smaller packet size is applied on DL, shorter frame size on the uplink (UL), multiuser packet on DL, and higher peak data rate on both DL and UL. The rate control algorithm on the UL plays a crucial role in enhancing the UL data rate. User data transmission is time division multiplexed on the DL, while being code division multiplexed on the UL. In EV-DO Rev.A, five fading channel models are adopted, which are Mode A-E [1]. Each of these fading channel models corresponds to a specific number of paths, path delay, power profile, and vehicular speed.

The 3rd Generation Partnership Project (3GPP) Release 5 and Release 6 extend WCDMA specification with High Speed Downlink Packet Access (HSDPA) and High Speed Uplink Packet Access (HSUPA) respectively [6, 7]. In HSDPA, several advanced features are adopted, which include adaptive modulation and coding (AMC) and Hybrid Automatic Repeat Request (HARQ). The multipath profiles of the fading channel used in WCDMA HSDPA/HSUPA, denoted by HSxPA, in this paper, are defined in [2], with Pedestrian A (PA) - 3km, Pedestrian B (PB) - 3km, Vehicular A (VA) - 30km and Vehicular $-120 \mathrm{~km}$ as the most representative models.

802.16e Mobile WiMAX, denoted by Mobile WiMAX for short thereinafter, is a newly emerging $3 \mathrm{G}$ candidate technology [8, 9]. It is based on OFDM and employs TDD. This is different from EV-DO Rev.A and HSxPA both of which are based on CDMA and use FDD. Mobile WiMAX is flexible in providing asymmetrical services and in making use of unpaired spectrum. AMC, HARQ and other techniques are also used in order to provide satisfactory data rate. For single transmission antenna scenario, mixed 6-finger multipath models are used, while for Multiple Input Multiple Output (MIMO) scenarios, Spatial Channel Model enhanced (SCMe) is usually used to generate channel parameters.

Table I presents a point by point comparison of key attributes. It is easily seen from the table that these technologies adopt different duplex modes as aforementioned.
TABLE I

SUMMARY OF MAIN ATtRIBUTES

\begin{tabular}{|c|c|c|c|}
\hline Attribute & EV-DO Rev.A & HSxPA & WiMAX \\
\hline Duplex mode & FDD & FDD & TDD \\
\hline Multiple access & $\begin{array}{l}\text { DL: TDMA } \\
\text { UL: CDMA }\end{array}$ & $\begin{array}{l}\text { DL: TDMA \& } \\
\text { CDMA } \\
\text { UL: CDMA }\end{array}$ & OFDMA \\
\hline $\begin{array}{l}\text { System band } \\
\text { width (MHz) }\end{array}$ & $2 * 1.25$ & $2 * 5$ & $\begin{array}{l}\text { Scalable, } \\
\text { e.g. } 5 \text { or } 10\end{array}$ \\
\hline Slot length (ms) & $\begin{array}{l}\text { DL: } 1.67 \\
\text { UL: } 6.67\end{array}$ & $\begin{array}{l}\text { DL: } 2 \\
\text { UL: } 2 \text { or } 10\end{array}$ & 5 (TDD) \\
\hline $\begin{array}{l}\text { Peak data rate } \\
\text { (Mbps) }\end{array}$ & $\begin{array}{l}\text { DL: } 3.1 \\
\text { UL: } 1.8\end{array}$ & $\begin{array}{l}\text { DL: } 14.4 \\
\text { UL: } 5.8\end{array}$ & $\begin{array}{l}\text { DL: } 46 \\
(\mathrm{DL}: \mathrm{UL}=3: 1) \text {; } \\
\text { UL: } 7 \\
(\mathrm{DL}: \mathrm{UL}=1: 3)\end{array}$ \\
\hline Modulation & $\begin{array}{l}\text { BPSK/QPSK } \\
\text { 8PSK/16QAM }\end{array}$ & $\begin{array}{l}\text { BPSK/QPSK } \\
\text { 16QAM }\end{array}$ & $\begin{array}{l}\text { QPSK/16QAM } \\
\text { 64QAM }\end{array}$ \\
\hline Coding & Turbo & Turbo or $\mathrm{CC}^{1}$ & Turbo or $\mathrm{CC}^{1}$ \\
\hline HARQ & Synchronous IR & $\begin{array}{l}\text { Asynchronous } \\
\text { CC }^{2}\end{array}$ & $\begin{array}{l}\text { Asynchronous } \\
\mathrm{CC}^{2}\end{array}$ \\
\hline $\begin{array}{l}\text { Maximum } \\
\text { retransmission }\end{array}$ & $\begin{array}{l}\text { DL: } 0,1,3,7,15 \\
\text { UL: } 3\end{array}$ & $\begin{array}{l}5 \text { for } 2 \mathrm{~ms} \text { TTI, } \\
3 \text { for } 10 \mathrm{~ms} \mathrm{TTI}\end{array}$ & 3 \\
\hline Handoff & Soft Handoff & $\begin{array}{l}\text { Network- } \\
\text { Initiated } \\
\text { Hard Handoff }\end{array}$ & $\begin{array}{l}\text { Network- } \\
\text { Optimized } \\
\text { Hard Handoff }\end{array}$ \\
\hline
\end{tabular}

${ }^{1}$ Convolution Code

2 Chase Combining

In addition, different bandwidths are specified. While some key techniques, such as HARQ, are employed in all these technologies, detailed configurations, such as retransmission number in HARQ, vary from one technology to another. Such configuration diversity makes it challenging to perform performance comparison between these technologies. In the next section, we present a framework for the comparison, where we refer to a network using one of the specific technologies described in this section as a system, such as EV-DO Rev.A system.

\section{A FRAMEWORK FOR COMPARISON}

As each technology has different operating parameters and algorithms, it is imperative for fair comparison that we should find a set of unified configurations. In this section we propose a simulation framework that includes a common channel model, operating parameters and algorithms.

\section{A. A Mixed Channel Model}

Since channel models play an important role in evaluating various system performances, 3GPP, 3GPP2 and International Telecommunication Union (ITU), have recommended a set of channel models that have been used for EV-DO Rev.A, HSxPA and Mobile WiMAX with SIMO antenna configuration (typically $1 \mathrm{Tx}$ and $2 \mathrm{Rx})^{3}$.

There is rich variety of channel models, for example, there are 5 channel models in 3GPP2. Moreover, the channel models suitable for wideband and narrowband systems are different because each system uses different technology, which has

\footnotetext{
${ }^{3}$ For reference, channel models adopted by 3GPP, 3GPP2, and WiMAX are summarized in Table 2.2.2-1 and Table 2.2.2-2 in [1], in Table A-3 to Table A-6 in [2] and in Table 16 and Table 17 in [10], respectively.
} 
different finger numbers and resolution. Thus we can see that a unified channel model is essential for fair comparison of the wireless systems. For instance, some works [4] adopted channel models which are more advantageous for a specific system because there is no unified channel model.

To tackle this problem, we propose the following mixed model: PB-3km, VA-30km and VA-120km with distribution probabilities of $60 \%, 30 \%$ and $10 \%$, respectively (detailed configurations can be found in [11] ). This mixed model is a common set of the models originally proposed for each technology, so all related parameters can be found in wellknown works $[1,2,10]$ and we believe it is reasonable enough as it considers the fading channel characteristics of the systems appropriately.

\section{B. Common Key Parameters}

As we have mentioned before, each system has a different set of parameters, among which some are configurable while others are hardware limited. Therefore, in order to fairly compare various performance indices of the systems, we have surveyed a number of relevant documents and managed to summarize a unified system configuration as shown in Table II, where the operating frequency is set to $2 \mathrm{GHz}, 1$-by-1 antenna is used on the downlink and 1-by-2 antenna is used on the uplink.

TABLE II

AN OVERALL SySTEM CONFIGURATION

\begin{tabular}{|c|c|c|}
\hline Simulation parameters & Unit & Values \\
\hline Operating frequency & $\mathrm{GHz}$ & 2 \\
\hline Log-normal std. deviation & $\mathrm{dB}$ & 8.9 \\
\hline Thermal noise density & $\mathrm{dBm} / \mathrm{Hz}$ & -174 \\
\hline Frequency reuse factor & & 1 \\
\hline BS-to-BS distance & $\mathrm{km}$ & 2 \\
\hline $\begin{array}{l}\text { Minimum distance } \\
\text { between BS and MS }\end{array}$ & $\mathrm{m}$ & 35 \\
\hline $\begin{array}{l}\text { Maximum UL total path } \\
\text { loss (no fading) }\end{array}$ & $\mathrm{dB}$ & 140 \\
\hline Antenna configuration & & DL: $1 * 1$ UL: $1 * 2$ \\
\hline Propagation model & & $\begin{array}{l}128.1+37.6 \cdot \log _{10} d \\
(\mathrm{~d} \text { in } \mathrm{km})\end{array}$ \\
\hline Antenna height of BS & $\mathrm{m}$ & 30 \\
\hline Antenna height of MS & $\mathrm{m}$ & 1.5 \\
\hline BTS Tx power & $\mathrm{dBm}$ & $\begin{array}{l}43 \text { per carrier in CDMA } \\
\text { systems; } 43 \text { per antenna in } \\
\text { WiMAX }\end{array}$ \\
\hline $\begin{array}{l}\text { BTS Tx/Rx antenna gain } \\
\text { with cable loss }\end{array}$ & $\mathrm{dBi}$ & 15 \\
\hline Other loss & $\mathrm{dB}$ & 10 \\
\hline BTS noise figure & $\mathrm{dB}$ & 5 \\
\hline Antenna horizontal pattern & $\mathrm{dB}$ & $\begin{array}{l}70 \mathrm{deg}(-3 \mathrm{~dB}) \text { with } 20 \mathrm{~dB} \\
\text { front-to-back ratio }\end{array}$ \\
\hline Correlation between BS & & 0.5 \\
\hline MS Tx power & $\mathrm{dBm}$ & $\begin{array}{l}23 \text { in EV-DO and WiMAX } \\
21 \text { in HSUPA }\end{array}$ \\
\hline MS Tx/Rx antenna gain & $\mathrm{dBi}$ & -1 \\
\hline MS noise figure & $\mathrm{dB}$ & 9 \\
\hline Other losses & $\mathrm{dB}$ & 10 \\
\hline
\end{tabular}

\section{Common Key Algorithms}

Regarding radio resource management algorithms that are essential to system performance, we have adopted most de- ployed algorithms [1-3, 12]. In addition, some important techniques are also used, such as HARQ and AMC. Compared with EV-DO Rev.A and HSxPA, the standardization progress of Mobile WiMAX is not as complete as the other two. But we have decided to give a brief description about the scheduling algorithm and dynamic overhead model in Mobile WiMAX, because they have great effect on the simulation results.

MAC scheduler related contents and detailed scheduling mechanisms are introduced in [3], where the scheduling process can be divided into several steps: (i) determination of packet transmission ordering over air interface, (ii) resource allocation and creation of DL/UL maps. In the meantime, a proportional fair algorithm used for multi-carrier systems is developed in [13]. In this paper, we regard the sub-channels in WiMAX as the multi-carriers in [13] to use the algorithm proposed in [13].

The physical overhead is a critical factor that significantly affects the overall performance. The overhead is usually set to a fixed value, i.e., 7 symbols on DL and 3 symbols on UL, in most public documents, such as in $[4,10]$. Though several works $[14,15]$ tried to consider the MAC overhead in Mobile WiMAX systems, more precise amount of the overhead size in the physical layer of Mobile WiMAX has recently been calculated in [16]. However, [16] still assumes fixed overhead size, which means the overhead size is predefined before the simulation and will not be changed according to dynamic number of users, scheduling decisions and other factors. In this paper, we use the dynamic overhead model that was proposed by us [17] to evaluate more accurate performances.

\section{Simulation Results}

In this section, we evaluate the system performances through extensive system-level simulations. Detailed descriptiong about simulation methodology can be found in [11].

\section{A. Platform Calibration}

The fairness curve is defined as the cumulative distribution function $(\mathrm{CDF})$ of the normalized user throughput, where the normalized user throughput is defined as follows:

$$
\overline{T_{i}}=\frac{T_{i}}{\frac{1}{N} \sum_{n=1}^{N} T_{n}} .
$$

Here $T_{i}$ is the throughput of user $i$, and $N$ represents the number of users. To decide whether a system is fair enough or not, we use three-points fairness criterion [1] which is met if a fairness curve of a system lies on the right side of the line segments defined by three points, i.e., $(0.1,0.1)$, $(0.2,0.2)$ and $(0.5,0.5)$. Though three-points criterion is not directly associated with well-established fairness criteria, e.g., proportional fairness, it has served as a handy graphical method in practical wireless systems which can decide whether a fairness curve is acceptable.

The fairness among all users can be achieved by using a proper scheduling algorithm, such as Proportional Fair (PF) scheduler [18]. In order to achieve satisfactory fairness and high spectral efficiency at the same time, we have calibrated 


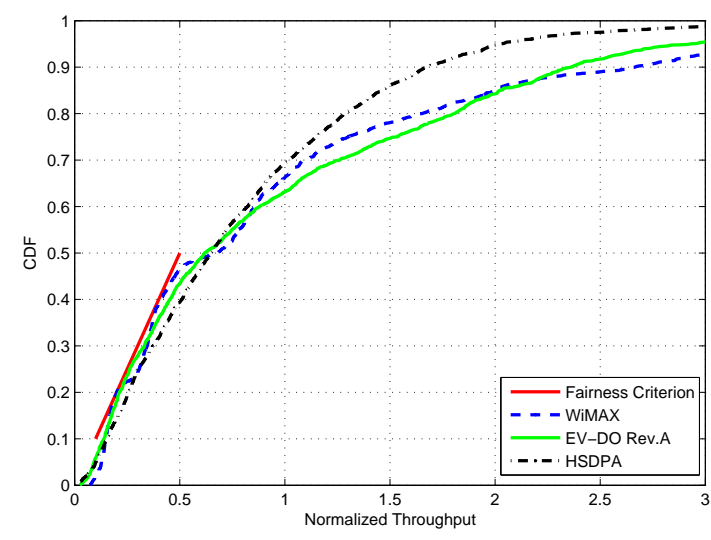

(a) Downlink

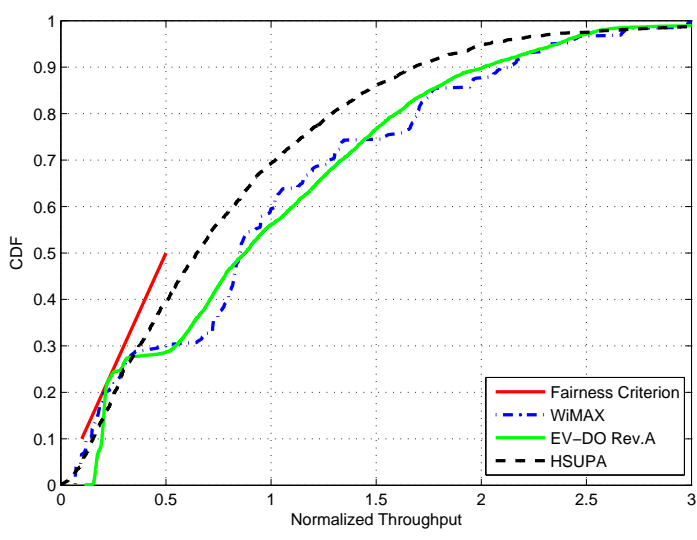

(b) Uplink

Fig. 1. Fairness curves of each system on DL/UL.

TABLE III

DL/UL THROUGHPUTS

\begin{tabular}{|c|c|c|c|}
\hline \multicolumn{4}{|c|}{ DL Throughput } \\
\hline \hline Users/Sector & WiMAX (DL:UL=2:1) & EV-DO & WCDMA HSDPA \\
\hline 4 & 2.412 & 1.009 & 3.036 \\
\hline 10 & 2.626 & 1.067 & 3.752 \\
\hline 16 & 2.690 & 1.074 & 3.931 \\
\hline \multicolumn{4}{|c|}{ UL Throughput } \\
\hline Users/Sector & WiMAX (DL:UL=2:1) & EV-DO & WCDMA HSUPA \\
\hline 4 & 1.130 & 0.673 & 1.403 \\
\hline 10 & 1.362 & 0.600 & 0.971 \\
\hline 16 & 1.510 & 0.471 & 0.520 \\
\hline
\end{tabular}

relevant parameters used in scheduling algorithms such that the following two criteria are met: i) fairness curves are similar to each other in shape, which will guarantee the consistence and fairness among the systems; ii) fairness curves are as close enough to the fairness criterion, which indicates that the throughput is maximized.

Fig. 1 shows the average fairness curves on the DL and UL with 10 users per sector respectively. It is shown that all curves are on the right side of the three-point fairness segments, which indicates fairness among users. At the same time, each curve is as close to the fairness criterion as possible, which means that the curve can not be shifted toward left side while not exceeding the criterion. In addition, the three curves are close to each other, which signifies they are similar to each other in terms of fairness.

\section{B. Throughput and System Spectral Efficiency}

Table III lists the sector throughputs of each system under different user densities (4, 10 and 16 users per sector). Note that the downlink to uplink time ratio is set to $2: 1$ in Mobile WiMAX, which is the typical setting for data service and can be compared with the other systems when only full buffer traffic users exist. It should be noted that HSDPA achieves the highest sector throughput on downlink, whereas Mobile WiMAX achieves the highest on uplink.

Since the system bandwidths are different, someone might prefer to compare system spectral efficiencies, in unit of $\mathrm{bit} / \mathrm{s} / \mathrm{Hz}$, which is defined as the amount of useful information that can be transmitted over per bandwidth unit per second. Since Mobile WiMAX is a TDD system, the notion of effective bandwidth is introduced to calculate the system spectral efficiency as shown in the following equations:

$$
\begin{gathered}
\lambda_{j}=\frac{T L_{j}}{B W_{j}^{\mathrm{eff}}}, \quad(j=0,1) \\
B W_{j}^{\mathrm{eff}}=B W \times \frac{\xi_{j}}{\sum_{j=0}^{1} \xi_{j}}
\end{gathered}
$$

where subscript $j$ represents downlink $(j=0)$ and uplink $(j=1)$, $T L_{j}$ denotes corresponding throughput, and $B W_{j}^{\text {eff }}$ is the effective bandwidth which is defined in (3). The transmit time ratio $\xi_{j}$ is 2 and 1 for downlink and uplink, respectively.

As shown in Table IV, Mobile WiMAX performs worst on downlink in terms of the system spectral efficiency. On the contrary, EV-DO Rev.A exhibits the best performance on DL and UL as well. It should be remarked that the uplink system spectral efficiency for the two CDMA-based systems decreases with the number of users. This is mainly due to the following reasons: i) more power is occupied to transmit the UL pilot; ii) more resource is used to maintain the minimal transmit rate for each user and more interference is suffered by each user. In Mobile WiMAX, however, the system spectral efficiency increases with the number of users owing to multiuser diversity.

\section{System Stability on the UL}

On the uplink, rise over thermal (RoT) and its outage rate are two important performance measures indicating the degree of system stability, which has significant impact on users' satisfaction in practical systems. RoT is defined as the ratio of total power (denoted by $I_{t}$ ) received at the base station to the power of thermal noise (denoted by $N_{0}$ ) shown in the following equation:

$$
R o T=\frac{I_{t}}{N_{0}}=\frac{I+N_{0}}{N_{0}},
$$


TABLE IV

DL/UL SySTEM SPECTRAL EFFICIENCIES

\begin{tabular}{|c|c|c|c|}
\hline \multicolumn{4}{|c|}{ DL System Spectral Efficiencies } \\
\hline \hline Users/Sector & WiMAX (DL:UL=2:1) & EV-DO & WCDMA HSDPA \\
\hline 4 & 0.3540 & 0.8074 & 0.6072 \\
\hline 10 & 0.3848 & 0.8539 & 0.7504 \\
\hline 16 & 0.3951 & 0.8590 & 0.7862 \\
\hline \hline \multicolumn{4}{|c|}{ UL System Spectral Efficiencies } \\
\hline \hline Users/Sector & WiMAX (DL:UL=2:1) & EV-DO & WCDMA HSUPA \\
\hline 4 & 0.3478 & 0.5385 & 0.2807 \\
\hline 10 & 0.4261 & 0.4798 & 0.1943 \\
\hline 16 & 0.4731 & 0.3770 & 0.1041 \\
\hline
\end{tabular}

where $I_{t}$ is divided into two part: power of thermal noise $N_{0}$ and power from all users $I$. We can see from (4) that higher RoT indicates more interference by assuming $N_{0}$ is a constant. Typically, RoT in decibel $(\mathrm{dB})$ is usually used.

RoT outage rate is defined as the probability that instantaneous RoT exceeds a threshold:

$$
P\left(R o T^{d B} \geq 7 d B\right) \leq 1 \%
$$

where $7 \mathrm{~dB}$ is commonly accepted as the outage threshold and $1 \%$ as the maximal outage rate. In addition, the stability criterion is said to be met if the condition (5) is satisfied. To satisfy the stability criterion in various wireless systems, we adjusted the RoT threshold used in uplink scheduling or the parameters used in rate control algorithms.

In practical wireless systems, Complimentary Cumulative Distribution Function (CCDF) has been used for performance analysis in extensive literature. To be more specific, the inflection point of $\mathrm{CCDF}$ indicates the approximate mean value and the steepness of CCDF implies the small variance. Wireless systems operators usually require that the mean value should be as large as possible and the variance should be as small as possible. The reason for the former statement is that larger average RoT indicates the system can work under higher interference level where high transmission rates (or more users for voice traffic) can be accommodated, because there exists efficient interference control mechanism to eliminate the negative influence caused by higher interference. As for the variance, larger variance means that RoT suffers from high fluctuation in different time slots, which on the other hand indicates that the interference level changes drastically and so as the signal strength. Therefore, the variance is predicted to be as small as possible.

Fig. 2(a) shows the CCDF of each system when the stability criterion is met. We can observe that the curve for EV-DO Rev.A is much steeper that the other two, which indicates smaller variance and better system stability. The other two curves are on the left of EV-DO Rev.A curve, and this signifies that the mean RoTs of Mobile WiMAX and HSUPA are smaller than that of EV-DO Rev.A (which is about 6.2 dB). Briefly speaking, EV-DO Rev.A can cope with higher interference level while keeping itself stable.

To further illustrate this observation, more results are shown in Fig. 2(b), where the mean RoTs of Mobile WiMAX and
HSUPA are adjusted to the same value with EV-DO Rev.A, i.e., $6.2 \mathrm{~dB}$. It is shown that the RoT outage rate considerably exceeds the threshold (The excess probability is about $30 \%$ ). In addition, the tail of the curve, which extends to more than $10 \mathrm{~dB}$, also indicates poor interference control mechanism.

The main reason for unstability lies in the initial design concept, where more flexibility was emphasized and therefore the scheduling scheme is open and depends on the operator's choice. In this paper, we adopted the most commonly used algorithms, which can be implemented easily. If there does exist complex algorithms which can substantially enhance the system spectral efficiency of these systems, there is room for ranking change. Therefore, we claim that there is a tradeoff between higher system spectral efficiency and deployment complexity for HSUPA and Mobile WiMAX. On the contrary, the 1-bit based rate control algorithm clearly specified in EVDO Rev.A is simple enough and can be implemented readily.

\section{Promising Techniques}

The results shown above are based on the framework presented in Section III. However, since virtual MIMO is regarded as one of potential technoliques which can considerably improve the performance of Mobile WiMAX, especially on its uplink, we try to be fair by showing its performance gain in this section. In the meantime, interference cancelation technique is being highlighted as a potential method to improve the performances of cdma systems. Fig. 3 compares the system spectral efficiency with these technologies, where there are 10 users per sector. It can be observed that the performance gain of EV-DO Rev.A is the highest, which is about $80 \%$ with system spectral efficiency of 0.9 bps per $\mathrm{Hz}$ per cell, whereas the performance gains of Mobile WiMAX and HSUPA are about $20 \%$ to $30 \%$. The perfect interference control looks to be the most promising technique which can lead to a dramatic improvement in EV-DO Rev.A.

\section{CONCLUSIONS}

In this paper, we presented a framework for performance comparison between three widely adopted technologies for $3 \mathrm{G}$ networks, which are CDMA2000, WCDMA and WiMAX.

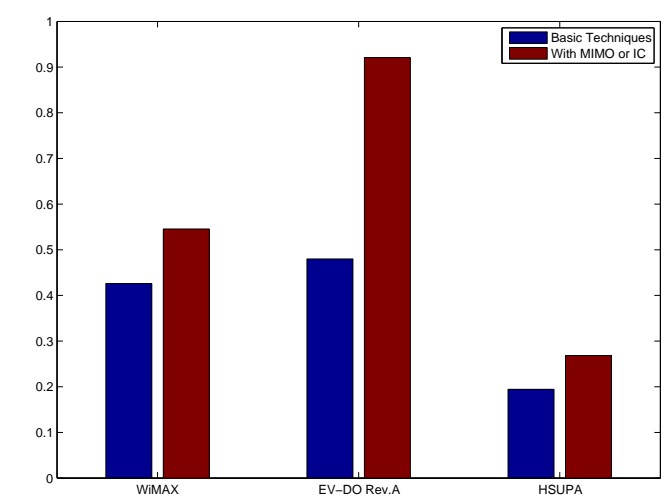

Fig. 3. System spectral efficiency comparison with enhanced techniques 


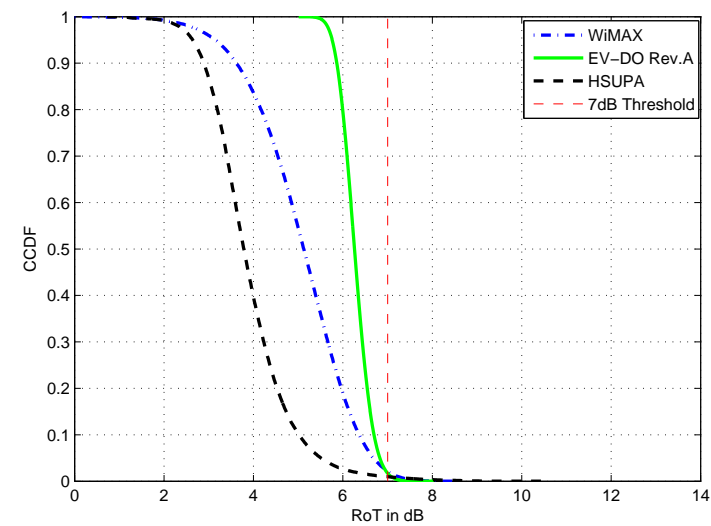

(a) When stability criterion is fulfilled

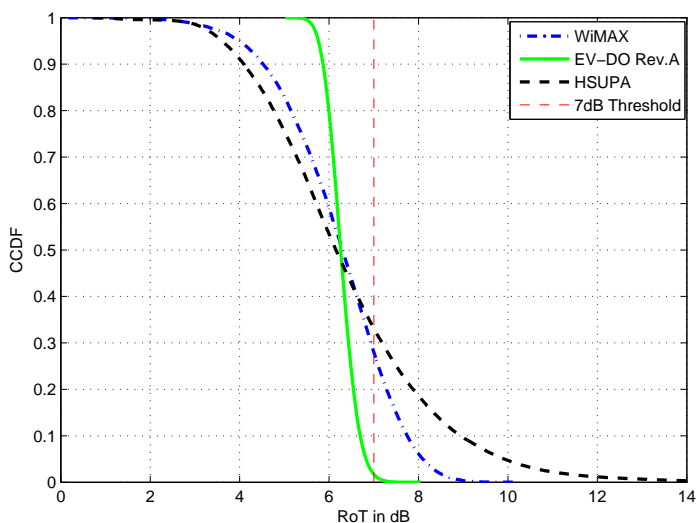

(b) When mean RoT is adjusted to $6.2 \mathrm{~dB}$

Fig. 2. CCDF curves of RoT with 10 users/sector.

Our first contribution lies in finding an identified common set of channel models, key algorithms and important parameter settings among these technologies. In addition, in order to give a fair comparison, special care has been taken: The simulation platform is calibrated so that the throughput is maximized and at the same time a fairness criterion among users is met for each technology. Simulation results have been compared, and our second key finding is that the outcomes in existing literatures are not the case if all technologies are compared in an less-biased manner. It is found, from our results, that while Mobile WiMAX gives higher throughput, it is not as good as EV-DO Rev.A and HSxPA in terms of system spectral efficiency especially on the downlink. Between EV-DO Rev.A and HSxPA, the system spectral efficiency of the former outperforms the latter. In addition, EV-DO Rev.A performs the best in terms of system stability, owing to the well standardized rate control algorithm on the uplink. For Mobile WiMAX and HSUPA, their interference control mechanism might need further improvement to provide better performance when the system could suffer from high interference. We believe our this work can serve as a cornerstone for a fair comparison between the three $3 \mathrm{G}$ technologies.

\section{REFERENCES}

[1] 3GPP2 C.R1002-A v0.1. cmda2000 Evaluation Methodology. March 2008.

[2] 3GPP TR25.896 v6.11.0. Feasibility Study for Enhanced Uplink for UTRA FDD. December 2006.

[3] WiMAX Forum. WiMAX System Evaluation Methodology. January 2007.

[4] WiMAX Forum. Mobile WiMAX - Part II: A Comparative Analysis. May 2006.

[5] 3GPP2 C.S0024-A. cdma2000 High Rate Packet Data Air Interface Specification. September 2006.

[6] 3GPP TR 25.808. FDD Enhanced Uplink: Physical Layer Aspects. March 2005.

[7] 3GPP TR 25.858. High Speed Downlink Packet Access: Physical Layer Aspects. March 2002.
[8] IEEE Std 802.16-2004. IEEE Standard for Local and metropolitan area networks: Part 16: Air Interface for Fixed Broadband Wireless Access Systems. 2004.

[9] IEEE Std 802.16e 2005. IEEE Std 802.16e-2005 and IEEE Std 802.16-2004-Cor 1-2005 (Amendment and Corrigendum to IEEE Std IEEE Std 802.16-2004). 2005.

[10] ITU Document 8F/1079-E. Additional Technical Details Supporting IP-OPDMA As an IMT-2000 Terrestrial Radio Interface. December 2006.

[11] Y. Gao, X. Zhang, D. Yang and Y. Jiang. Unified Simulation Evaluation for Mobile Broadband Technologies. IEEE Communication Magzine, March 2009.

[12] 3GPP TS25.214 v8.0.0. Physical layer procedures (FDD). December 2006.

[13] H. Kim and Y. Han. A Proportional Fair Scheduling for Multicarrier Transmission Systems. IEEE Communications Letters, 9:210 - 212, March 2005.

[14] M. Settembre, M. Puleri, S. Garritano, et al. Performance Analysis of an Efficient Packet-Based IEEE 802.16 MAC Supporting Adaptive Modulation and Coding. In 2006 International Symposium on Computer Networks, pages 11 - 16, June 2006.

[15] H. Lee, T. Kwon, D. Cho, et al. Performance Analysis of Scheduling Algorithms for VoIP Services in IEEE 802.16e Systems. In IEEE 63rd Vehicular Technology Conference, volume 3, pages 1231 - 1235, 2006.

[16] D. Chen. On the Analysis of Using 802.16e WiMAX for Point-to-Point Wireless Backhaul. In IEEE Radio and Wireless Symposium 2007, pages 507 - 510, January 2007.

[17] Y. Gao, L. Chen, X. Zhang, et al. Performance Evaluation of Mobile WiMAX with Dynamic Overhead. In IEEE 68th Vehicular Technology Conference, 2008.

[18] P. Viswanath, D. N. C. Tse, and R. Laroia. Opportunistic beamforming using dumb antennas. IEEE Trans. Inform. Theory, 48:1277-1294, June 2002. 